\title{
Are cytokines our key to immunity against mycobacterial diseases?
}

\author{
ROSEMARY WEIR \\ London School of Hygiene and Tropical Medicine, Keppel Street, \\ London WCIE 7HT, UK
}

A current dynamic area of mycobacterial research is the identification of correlates of protective immunity to mycobacteria. Such a correlate (either in vivo or in vitro) would greatly enhance our understanding of the pathogenesis of both leprosy and tuberculosis; would assist in the identification and evaluation of promising vaccine candidates; and may provide a more accurate marker of exposure than do the current skin tests. In this context, there are an increasing number of studies being conducted, such as that recently reported by Lima et al., ${ }^{1}$ that are measuring in vitro cytokine responses-from blood cells stimulated with mycobacterial proteins - as the 'read out' of the immune response in an individual. Findings to date from these studies indicate that cytokine responses-most prominently the interferon-gamma (IFN $\gamma$ ) response-may indeed provide useful markers of immune response that could be employed as correlates of protective immunity, although further evaluation is required. IFN $\gamma$ production by $\mathrm{T}$-cells is a key component of the protective response against mycobacteria, as it activates infected macrophages to kill intracellular M. leprae or M. tuberculosis.

It has been recognized that current basic laboratory research on leprosy can benefit from the intense efforts underway to define the protective immune response against tuberculosis, and-given our experience with BCG-it may be that a new more effective tuberculosis vaccine could also be effective against leprosy. BCG vaccines have shown widely varying protective efficacy against pulmonary tuberculosis depending on the area of the world in which they have been used. ${ }^{2}$ In the course of TB protection studies, it emerged that $\mathrm{BCG}$ is also highly protective against leprosy ${ }^{3}$ although this may also vary according to location. ${ }^{4}$

The development of the human immune response to BCG vaccination is being studied by several groups. Ravn et al. ${ }^{5}$ found that in 20 healthy Danish donors vaccinated with BCG (Copenhagen strain), in vitro peripheral blood mononuclear cell (PBMC) proliferative responses to tuberculin PPD and fractions/secreted proteins of $M$. tuberculosis increased more rapidly during the year following vaccination in those subjects who had responded to M. tuberculosis PPD by in vitro assay prior to BCG vaccination. They interpreted this observation as evidence that prior exposure to mycobacteria in these subjects may have played a role in priming the immune system. Interestingly, some of these 'primed' donors had 'negative' tuberculin skin test results. The IFN $\gamma$ response to secreted proteins was increased 2 months after vaccination in all donors, as was the cytotoxic response to PPD-pulsed macrophages, and tuberculin skin test sensitivity increased in the majority of 
donors. BCG vaccination has been shown to be highly protective against TB in Denmark. ${ }^{2}$ In contrast, a study of PBMC responses to tuberculin PPD and heat killed $M$. tuberculosis in a group of 20 tuberculin negative BCG vaccinees in South India found no increase in IFN $\gamma$ responses to PPD or heat-killed $M$. tuberculosis 2 months after BCG vaccination (Copenhagen strain), although tuberculin skin test sensitivity had increased. ${ }^{6}$ BCG vaccination has been shown to provide no protection against tuberculosis in South India. ${ }^{7}$ This suggests that the in vitro IFN $\gamma$ response may be a better correlate of BCG-induced protection against tuberculosis than the tuberculin skin test response. Lima et al. ${ }^{1}$ in Brazil have extended this work to analyse the BCG-induced protective response against leprosy. Their study of BCG vaccination (Moreau strain) in seven healthy household contacts of multibacillary leprosy patients (five of whom had previously received BCG in infancy) found that the IFN $\gamma$ responses to whole $M$. leprae in some of these subjects was increased 15 days and 1 year after vaccination with BCG. This was accompanied by a clear shift in the profile of the innate inflammatory response in all subjects - a decrease in the ratio of tumour necrosis factor $\alpha$ to interleukin 10 production. Although only small groups were investigated in each of these studies, they provide an indication of changes in cytokine response that result from BCG vaccination, which may reveal the mechanism behind development of a protective response against leprosy and/or tuberculosis, and therefore provide a tool to examine why BCG may not work in some populations. It is hoped that future larger scale studies of the immune response to. BCG vaccination, such as the parallel studies currently underway in Malawi and the UK (Black, Weir et al., manuscript in preparation), may further illuminate the important role of cytokines in mycobacterial infections.

\section{References}

${ }^{1}$ Lima MCBS, Pereira GMB, Rumjanek FD et al. Immunological cytokine correlates of protective immunity and pathogenesis in leprosy. Scand J Immunol, 2000; 51:419-428.

2 Fine PEM. Variation in protection by BCG: implications of and for heterologous immunity. Lancet, 1995; 346: 1339-1345.

${ }^{3}$ Karonga Prevention Trial Group. Randomized controlled trial of single BCG, repeated BCG, or combined BCG and killed Mycobacterium le prae vaccine for prevention of leprosy and tuberculosis in Malawi. Lancet, 1996; 348: 17-24.

${ }^{4}$ Fine PEM. South Indian leprosy vaccine trial: important lessons for mycobacterial immunology. Lepr Rev, 1999; 70: 247-249.

5 Ravn P, Boesen H, Pedersen BK, Andersen P. Human T cell responses induced by vaccination with Mycobacterium bovis Bacillus Calmette-Guerin. J Immunol, 1997; 158: 1949-1955.

6 Das SD, Narayanan PR, Kolappan C, Colston MJ. The cytokine response to Bacille Calmette Guerin vaccination in South India. Int J Tuberc Lung Dis, 1998; 2: 836-843.

7 Tuberculosis Prevention Trial Madras. Trial of BCG vaccines in South India for tuberculosis prevention. Ind J Med Res, 1980; 72: 1-74. 